\title{
An Open Source Software Quality Model and Its Applicability for Assessing E-commerce Content Management Systems
}

\author{
Wahyudi Agustiono \\ University of Trunojoyo Madura \\ Bangkalan, Indonesia \\ wahyudi.agustiono@trunojoyo.ac.id
}

\begin{abstract}
Content Management Systems (CMS) are widely known to provide a simple and practical way to create and administer a web application including e-commerce. A variety of CMS s exist which offer different features needed to develop an e-commerce. Nonetheless, there is limited knowledge on what framework can guide the selection of appropriate CMS for building a quality e-commerce. This is timely since ecommerce has been inhabited by the fears and risks associated with the online business. Five CMS e-commerce (WP ecommerce, Woocommerce, VirtueMart, Prestashop, and OpenCart) were chosen, deployed, customised, hosted in an online server as case studies of e-commerce and then tested against the quality criteria and metrics. This study provided empirical evidence on the applicability of the proposed model for gaining insights into the quality of e-commerce CMS.
\end{abstract}

Keywords-Software quality model; Open Source Software; Content Management System; E-commerce

\section{INTRODUCTION}

In the last few years, e-commerce has been one of the major investments for business to gain competitive advantages such as reducing cost, expanding the market, and providing better services [1]. It is, therefore not surprising; many modern businesses are interested and motivated to adopt e-commerce. Nonetheless, in reality, setting up e-commerce is likely difficult endeavour before it could be fully implemented. Especially for newly established or small businesses whose core business is not IT based; there are obstacles to be overcome in developing such e-commerce. The literature has indicated that a shortage of skilled labour, technical issues and fear of involving additional cost for hardware or software investment are among the challenges that should be addressed [2, 3].

Apart from that, there are lots more challenges involved in running the newly established e-commerce which require priority attention. This is because running e-commerce is likely much more than just about developing or having an online website. But beyond that, there are such vast arrays of socio-technical considerations. Examples include maintaining the system, ensuring the security, monitoring the website's traffics, retaining costumers' loyalty as well as building their trust in terms of quality of products, payment options and data privacy put on the web [4]. Therefore, failure in managing all of these issues properly can stop the e-commerce operation or even threaten the achievement of the goals.

With the current advancement in the web development approach using a Content Management System (CMS), the burden in building and sustaining an e-commerce likely can be minimised. This is because CMS provides all the features required to develop, set up an e-commerce and managing its content practically without the support from experts [5]. Additionally, since almost all of the CMSs are distributed under the open source platform, business will have the freedom to obtain, run, use and adapt them for developing ecommerce site without being charged licensing fee. Within the freedom principles, every CMS also comes with a community where anyone including businesses could either provide or seek necessary supports, as sistance and feedback from others regarding particular issues.

All these benefits have made CMS become the most popular tool that offers conformity in building an ecommerce, yet powerful. According to statistics by Digital Guide [6], there exist numerous between 250 and $350 \mathrm{CMSs}$ currently available in the community as standard solutions for professional web-store development. Nonetheless, not all CMSs are suitable to optimally support every business process. This is because CMSs comes with different variants and capability from a simple content to a complex framework for establishing an enterprise-wide system. Even if some CMSs have similar features, each of which however has its own characteristics, benefits, drawbacks and particular technical requirements in the implementation, installation, and configuration processes.

Based on the discussion above, it is clear that to identify and select an appropriate CMS for building a quality ecommerce is likely uneasy, especially for newly established or small businesses. Despite this challenge, there is limited knowledge on what quality model can be used to help businesses identify and select appropriate CMS that fits with their needs. Considering of this gap, this study aims to present quality model derived from existing standards which can be used for assessing CMSs. It then used the model to 
assess the quality of e-commerce CMSs as empirical evidence.

\section{LITERATURE REVIEW}

\section{A. Software Quality Models}

One of central discussion in software development since the early time is how to build quality software. According to ISO 25010, the quality of the software is defined as the degree to which the system satisfies the stated and implied needs of its various stakeholders and thus provides value [7]. According to Behkamal-Kahani [8] ensuring adequate the quality of the software can be only achieved by defining appropriate quality characteristics

This, in particular, has triggered practitioners and academia such as McCall, Boehm, Grady and HewlettPackard Co to provide models that are useful for assessing software quality [9]. The need for software quality assurance has gained more and more attention due to demand to build software product which conforms to particular requirements. In addition, the increasing number of failures and delays in a software project has also fuelled the need to develop more quality models [10]. Since the models are made up of a different combination of attributes and rooted from diverse area, very often they make the confusion that potentially resulting in an unexpected or imprecise output.

An initiative was taken by the International Organization for Standardisation (ISO) to develop consensus for standardised software quality attributes and metrics. An ISO/IEC 9126 was established in 1985 which measured the software quality form three perspectives: internal, external and quality in use. In 2010, ISO replaced 9126 standards with ISO 15010 which extends the model by including computer systems, and quality in use from a system perspective [7] as depicted in Fig 1. One common criticism with this standard is that it makes no consideration or provision for evaluating Open Source Software (OSS) quality. Furthermore, as the standard is intended for commercial software, the attributes and the metrics did not factor in a unique characteristic of OSS such as freedom principles [11].

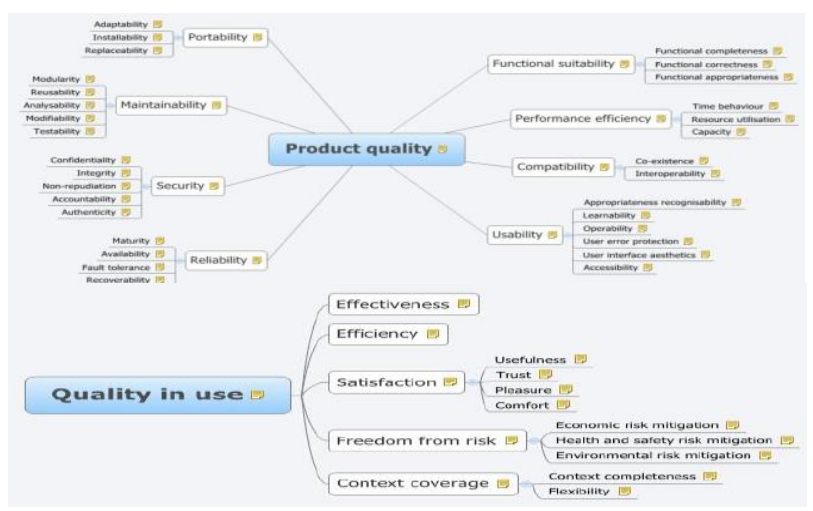

Fig. 1. ISO 25010 quality model
Review from the literature indicated a number of initiatives have been taken to define a quality model for OSS. Most of which are adapted from the ISO 9126 quality model with additional aspects on freedom principles of OSS [12]. However, the overall models provide generic quality attributes for OSS in general and make little consensus on the metrics used in the assessment. As a result, given the diverse nature of OSS packages, it is often difficult to apply the model for specific cases such as for assessing ecommerce Content Management System (CMS). Therefore, a more study that proposes a quality model and then demonstrates its applicability in evaluating e-commerce CMS quality in an empirical context is a warrant.

\section{B. E-commerce CMS quality model}

A CMS is defined as a database of information and a way to change and display that information, without spending a lot of time dealing with the technical details of presentation[13]. Using CMS, users without programming knowledge can create, edit, and publish web content, such as text or multimedia elements, collectively via a graphical user interface [6]. This conformity combined with the ease of use has clearly offered advantages and made CMS as most preferred choice especially for those a seeking practical way to build a web application.

Despite its popularity, CMS comes with lots of number of variances which on the other hand rise a major is sues on how to discover the best suit one. The difficulty is likely to multiply since there are limited knowledge and no single criteria for guiding the selection of the CMS [14]. A number of studies have attempted to identify and examine the existing quality model for OSS $[11,15]$. Other researchers have adopted and used the quality attributes and metrics provided by existing models for assessing the quality of open source software [16, 17].

However, in the era of digital transformation where individual or organisation seem to make their business go online, researches on examining the quality of CMS especially used for developing e-commerce are still lacking. One exception [18] found which compared three ecommerce CMSs. However, the authors did make clear what quality model used in the study. With regards to the gaps discussed above, this study therefore aimed at presenting quality model derived from existing standards which can be used for assessing e-commerce CMS. To demonstrate the usefulness of the model, it was then used for gaining insights into the quality of some e-commerce CMSs in an empirical setting.

\section{RESEARCH APPROACH}

This study applied two research strategies lasted nearly six months to address the research problem. Firstly, a quality model for assessing e-commerce was proposed derived from existing criteria. Secondly, this study then employed the model to assess five e-commerce CMSs to demonstrate its usefulness as discussed below. 


\section{A. Defining quality model of e-commerce CMS}

This study adopted the ISO 25010 model (see Fig 1) as an overarching framework that guided the development of e-commerce quality model. The ISO 25010 was chosen because this is most widely adopted series of quality standards. It also has great influence to the existing quality models in practices including the majority of OSS quality models that exist today $[11,12]$. Nonetheless, not all of the characteristics are likely applicable for measuring the quality of all types of software products including ecommerce CMS packages as pointed out in the previous critiques. For instance, health and safety risk mitigation characteristic is usually more applicable for assessing the quality of human-computer system rather than software product including e-commerce application. For this reason, this study did not include this sub-characteristic as the measurement.

Through checking the list of ISO 25010 high-level quality characteristics and related sub-characteristics only some parts are included in this study due to their relevancy on the open source software principles (e.g. freedom to use, adapt, modify and share). More specifically to enable the quality assessment, a number of methods were proposed in Table 1 below.

TABLE I. E-COMMERCE CMSEVALUATION MODEL

\begin{tabular}{|l|l|l|}
\hline $\begin{array}{l}\text { Quality } \\
\text { requirements }\end{array}$ & $\begin{array}{l}\text { Quality } \\
\text { Characteristics }\end{array}$ & $\begin{array}{l}\text { Testing strategies (approach, } \\
\text { method or tool) }\end{array}$ \\
\hline $\begin{array}{l}\text { Freedom } \\
\text { principles }\end{array}$ & $\begin{array}{l}\text { Economic risk } \\
\text { mitigation }\end{array}$ & Online checker: Google Trend \\
\hline \multirow{2}{*}{$\begin{array}{l}\text { Ease of } \\
\text { deployment }\end{array}$} & Compatibility & Dynamic Testing \\
\cline { 2 - 3 } & Portability & Blackbox testing: browsers \\
\cline { 2 - 3 } & Maintainability & Dynamic Testing \\
\hline \multirow{2}{*}{$\begin{array}{l}\text { System } \\
\text { performance }\end{array}$} & Reliability & $\begin{array}{l}\text { Online Stress testing: Webserver } \\
\text { Stress Tool (WAPT 8.1.) }\end{array}$ \\
\cline { 2 - 3 } & Efficiency & $\begin{array}{l}\text { Online web scanner: Pingdom } \\
\text { Tool }\end{array}$ \\
\hline $\begin{array}{l}\text { Control of } \\
\text { access }\end{array}$ & Security & $\begin{array}{l}\text { Software as a Service (SaaS): } \\
\text { Sucuri Sitecheck, white box and } \\
\text { dynamic testing }\end{array}$ \\
\hline
\end{tabular}

\section{B. Running quality assessment using proposed model}

Following the development of the quality model, the next step was running quality as ses sment as suggested in the previous work [19]. At this stage, Five CMS e-commerce (WP e-commerce, Woocommerce, VirtueMart Prestashop and OpenCart) were chosen, deployed, customised, hosted in an online server as case studies of e-commerce and then tested against the quality criteria defined in Table 1. During this phase, the key quality attributes of the five e-commerce websites were examined using the following approaches:

- White Box testing (Internal inspection) was done by analysing the software properties including the documentation, manual, source code, database and features that have an impact on software behaviour.

- Dynamic and Black box testing (External inspection) was done by either using software or executing the software and then measuring the dynamic attributes such as related to the behaviour of certain function and number of faults present in the program.

- Quality in use inspection was done similarly with external inspection but in a realistic context of use such as how users carrying out specific tasks

\section{RESULTS AND ANALYSIS}

To start assess e-commerce CMS quality, a number of ecommerce CMS candidates was identified. Following the "Generally Recognized as Mature" (GRAM) principles [20], this study chose five e-commerce CMS and set up these into fully functioning online store applications (see Table II). Using the set criteria defined in Section III, all the applications were then evaluated in-depth.

\section{TABLE II. FIVE E-COMMERCE CMS}

\begin{tabular}{|l|l|l|}
\hline No & E-commerce CMS & \multicolumn{1}{c|}{ Hosted URL } \\
\hline 1 & WP e-commerce & http://istanasport.16mb.com/ \\
\hline 2 & Woocommerce & http://www.woocom.16mb.com/ \\
\hline 3 & VirtueMart & http://joomla-dev.hol.es/ \\
\hline 4 & Prestashop & http://gayaku.16mb.com \\
\hline 5 & OpenCart & elektronikshop.000webhostapp.com \\
\hline
\end{tabular}

\section{A. Freedom principles}

One preliminary step prior to deploying an OSS is to ensure that the software complies with the freedom principle. Using the proposed model above, this corresponds to the economic risk mitigation quality characteristic that measures the ability of a software product in mitigating "the potential risk to financial status, efficient operation, commercial property, reputation or other resources in the intended contexts of use" [7]. This requirement, in particular, is reflected in open source licenses that allow the software to be freely obtained, used, distributed and modified at no cost.

Therefore, a basic way to evaluate the economic risk that arises from the OSS use is to read and review the license prior to installation. The review indicates that all five CMS packages investigated in this study use the GNU General Public License (GPL) that gives the freedom to copy, redistribute, understand, and modify the program. This finding, while primarily, suggests that all the software meet the quality requirement of e-commerce CMS.

Unfortunately, this basic assessment does not offer indepth into the quality of the software which later could provide practical knowledge for selecting the appropriate ecommerce CMS. One way is to read the comments and reviews of the online support forum where problems and issues are discussed and resolved by OSS developers and other users. Therefore, the more popular forum is, the more likely the OSS offers fewer risks. This is because a popular support forum makes it likely easy for users to notice and resolve problems associated with the deployment process. 


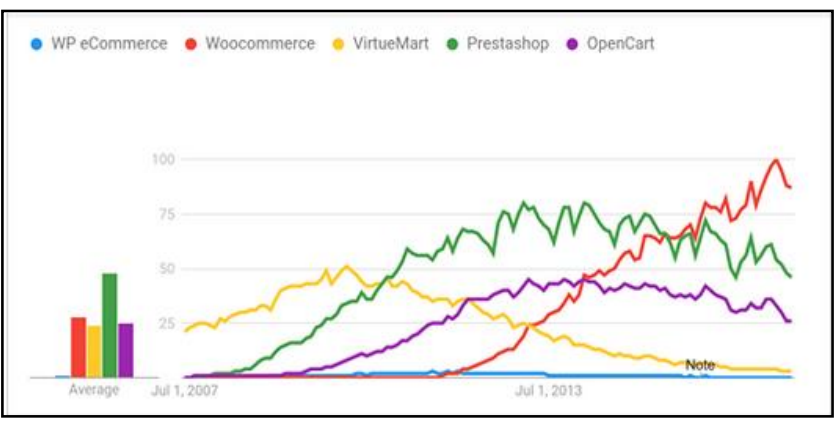

Fig. 2. Trends of five e-commerce CMS from 2007 to 2017

Nonetheless, reading and review support forums to compare and identify a quality OSS can be quite timeconsuming. There are some measurements tools that can help compare different product being considered. An example is Google Trend which could provide a useful metric of and help determine whether a product is increasing or decreasing in popularity. Figure 2 illustrates the popularity and trendiness of five OSS using Google Trend in the last ten years that shows the most popular CMS is Prestashop then followed by Woocommerce, OpenCart, VirtueMart and WP Commerce respectively. In general, this means that overall Prestashop offers better quality assurance in risk mitigation compared to other CMSs.

Another interesting finding is that in the last five years, Woocommerce is the only CMS that has gained positive trend of increasing its popularity. This finding suggests that those who are looking for better CMS to develop their online store can use Woocommerce. This is contrary to what VirtueMart that has experienced declining its popularity consistently. This observed decline in popularity could be related to the difficulties encountered by users in getting support from the forum to fix their problems themselves when deploying the CMS. This declining may be partly related to the developers' approaches which tend to make the users to pay especially for higher and complex technical supports instead of the online forum. Finally, Figure 2 also shows an unexpected finding of the very low popularity of WP commerce or even zero. It is difficult to explain this finding, but it might be related to the decision of the developer not to allow their forums to be indexed by Google which then affects to the result of the Google Trend.

\section{B. Ease of deployment}

Despite the freedom principle, the other reason why users need to consider before adopting the CMS is the ease of deployment especially when it comes to installation, set up configuration and applying related modules [21]. Based on the model above, this requirement positively affects the quality of OSS and can be assessed in depth using some criteria as discussed below.

\section{1) Compatibility}

ISO 25010 defines portability as "the degree of effectiveness and efficiency with which a system, product or component can be transferred from one hardware, software or other operational or usage environment to another"[7]. The objective of portability assessment is to see if the software can be successfully installed in a specified environment (installability). For this purpose, all five CMSs were installed in both local computer and web hosting account and then verified to see whether installation under different environment condition was free from failure. The investigation showed that all the CMSs can be installed successfully both on the local server and hosting service (see Table II).

\section{2) Portability}

The objective of portability assessment is to see how the software can be "adapted for different specified environments without applying components, actions or means other than those provided" [7]. For this purpose, each of the newly-installed CMSs was then set-up into a fully functional e-commerce store by running basic configuration, customising the theme and installing add-on modules required for the suitability and operability of an online store (e.g. payment, shipping and logistics). Finally, to see the portability performance, a different type of browsers (chrome, IE and Mozilla) were used to test the consistency of the website. Results indicated all the website can be best viewed in all browser platforms without failure except $\mathrm{Wp}$ e-commerce where the slider did not work well.

\section{3) Maintainability}

Maintainability assessment is aimed at defining how easy a software product can be modified for enhancements, corrective problems and adapting to changing needs. According to ISO 25010, the modification can include updating or upgrading the existing system (modifiability); module correction or even developing own component to suit with specific needs (modularity). One way employed in this study to determine the level of maintainability was by using a dynamic test that aimed to determine the ability of the forms interfaces in handling error detection and recovery. Analysis from dynamic testings showed all the form entries provided by the CMSs have error detection and recovery mechanisms such as highlighted incorrect entries, data consistency validation and feedback messages for further correction. Figure 3 shows an example of error detection and recovery mechanism.

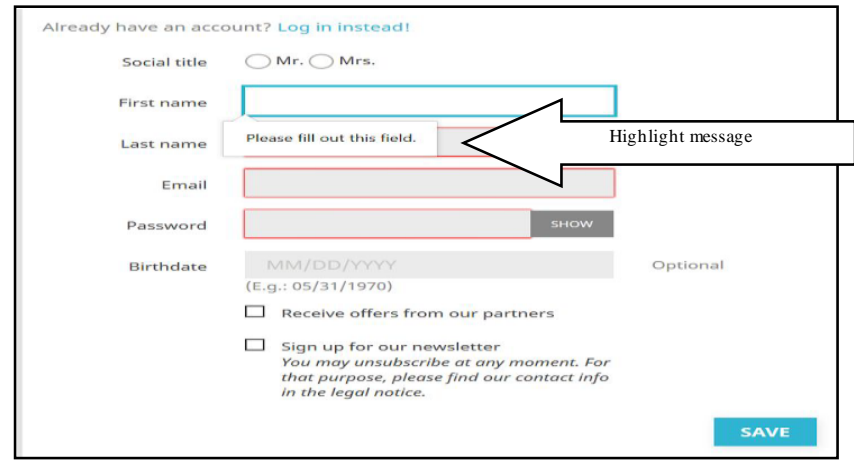

Fig. 3. Result on error prevention \& recovery mechanisms testing

\section{System Performance}

System performance is considered as the capability of the CMS accomplishes its designated functions within various constraints such as speed, accuracy, and 
completeness [7]. As proposed in Table 1, two quality attribute will be checked to determine the performance level. First, each CMS was checked for its reliability associated with the number of failures occurred in specific time, number of defects which could cause incorrect operation and data loss. For this purpose, stress testing approach was employed to identify faults using the Web Stress Tool (WAPT 8.1). Table 3 summarises the results of the web stress tool of five CMSs run in 10 minutes involving 20 users. The evaluation was repeated in the next day to provide more accurate results. It can be seen from the Table 3, WP, Woocommerce, and VirtueMart are considered to have excellent reliability. Whereas Prestashop and OpenCart have reliability issues especially Prestashop.

TABLE III. RESULT FROM WEB STRESS TOOL (WAPT 8.1)

\begin{tabular}{|l|l|l|l|l|}
\hline No & \multirow{2}{*}{ E-commerce CMS } & \multicolumn{2}{|c|}{ Number of errors } & \multirow{2}{*}{ Average } \\
\cline { 3 - 4 } & & Day 1 & \multicolumn{1}{|c|}{ Day 2 } & \\
\hline 1 & WP e-commerce & 0 & 0 & 0 \\
\hline 2 & Woocommerce & 0 & 0 & 0 \\
\hline 3 & VirtueMart & 0 & 0 & 0 \\
\hline 4 & Prestashop & 101 & 100 & 100 \\
\hline 5 & OpenCart & 2 & 78 & 40 \\
\hline
\end{tabular}

Second, this study also needed to as sess the efficiency of each CMS. According to ISO 25010, the efficiency is considered as the number of resources required by each CMS to perform a specific function. For this purpose, Pingdom (https://www.pingdom.com/), an online website scanner services, was used to help gain instant and real-time insights into each CMS' performance. Analysis from the performance testing using Pingdom indicated that almost all CMSs' performances are considered as grade B (Good) except Prestashop that was found to be poor with grade D $(67 \%)$.

This unexpected finding could be attributed by the slow load time of the website. Another possible explanation for this might be the poor service of Leverage Browser Caching with grade $\mathrm{F}$ as shown in Figure 4. This service enables a browser to store all static files required to load a website. Therefore, with such poor or unavailable cache services, the users' browser would download and retrieve all the files again from the servers which in particular made the loading speed longer.

\section{Control of access}

Another important quality attribute for a e-commerce CMS is security. ISO 25010 defines this as the ability of the system to provide required functionalities that ensure confidentiality, prevent unauthorised access to, or modification of and protect data and information. As mentioned in Table 1, two approaches were employed in this study to check the security level. First, using online SaaS, called Sucuri SiteChek, each CMS web-store was scanned to identify various security risks, vulnerabilities and possible threats that can potentially harm or damage both the system and data. Examples included malware, anomaly injection, website blacklisting and Outdated.

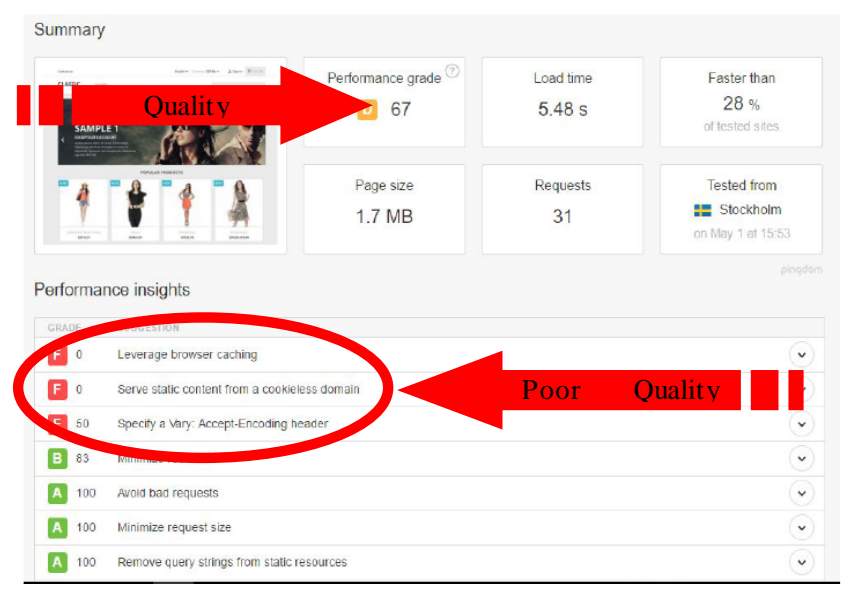

Fig. 4.Example of realtime perfomance test using pingdom

Table IV summarised the result of security test using Sucuri SiteChek. As can be seen from Table, it is clear that the web-store generated using OpenCart CMS has the lowest security risk. In the other hand, based on the test the web-store developed using PrestaShop and WP e-commerce was potentially to have security issues on web blacklisting and outdated respectively. Another interesting finding was that almost all CMSs except OpenCart were detected to have an issue on website firewall security. This finding suggests that the need to provide additional layer of protection when developing web-store using those CMSs to ensure to prevent destructive forces and threats.

TABLE IV. SECURITY ISSUES

\begin{tabular}{|l|c|c|c|c|c|}
\hline \multirow{2}{*}{ Security issues } & \multicolumn{5}{|c|}{ CMS Commerce } \\
\cline { 2 - 6 } & WP & Woo & Vir & Pres & Open \\
\hline Malware & Low & Low & Low & Low & Low \\
\hline Web Blacklisting & Low & Low & Low & High & Low \\
\hline Website Firewall & Med & Med & Med & Med & Low \\
\hline Website Outdated & High & Low & Low & Low & Low \\
\hline Injected spam & Low & Low & Low & Low & Low \\
\hline defacement & Low & Low & Low & Low & Low \\
\hline
\end{tabular}

Despite using SaaS to scan the overall security issues, this study also needs to examine the data security. This is critical as the e-commerce is supposed to have the ability in ensuring confidentiality, preventing illegal access to, or modification of and protect data and information using various techniques. One technique was using white box testing in which the each CMS's DBMS was examined in term of relational, performing query (add data, insert data, update data, select data, view data and delete data) for each table and check data structure consistency. To test the data security, functional testing was conducted to examine form interfaces using various data training. 


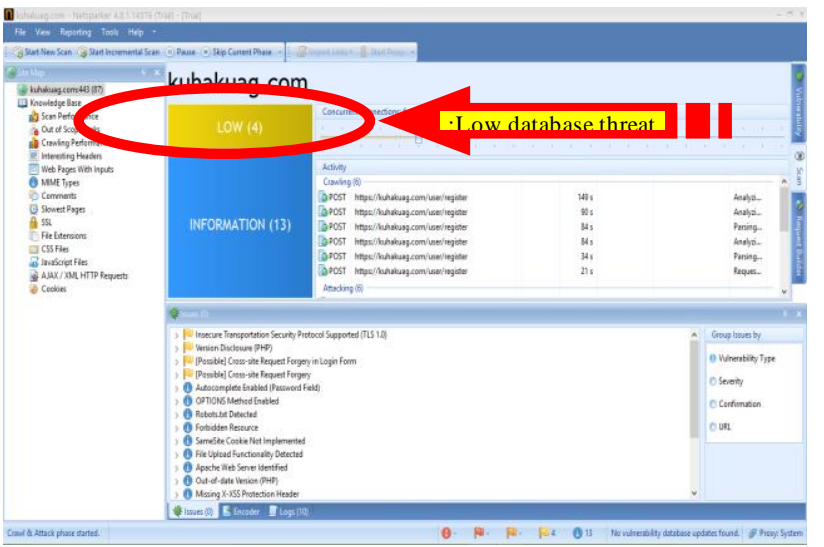

Fig. 5. Sample of SQL injection testing result

Finally, another way to examine the data security was by performing dynamic testing using SQL Injection (SQI) technique. SQLI is considered as one of simple and most common web hacking techniques yet it could destroy the database. To run this testing, a sample of malicious SQL queries, String \# and String 1'OR'1'='1 against a database server was injected into username and password of login form respectively. SQLI test was also conducted using NetSparker, a web application security, and vulnerability scanner to identify flaws in the database. Results indicated that the threat levels were rated as low as seen in Fig 5.

\section{CONCLUSION}

The objective of this study is to present quality criteria and metrics derived from existing models for assessing CMS e-commerce as seen in Table 1. Using the proposed model, five CMS e-commerce (WP e-commerce, Woocommerce, VirtueMart, Prestashop and OpenCart) were chosen, deployed, customised, hosted in an online server as case studies of e-commerce and then tested against the quality criteria and metrics. The overall finding was that this study has been able to demonstrate the usefulness of the proposed model, in guiding the assessment of CMS ecommerce. Therefore, this study made an important contribution to the existing knowledge by offering a quality model form which other researchers could use, adapt or extend the model for assessing OSS and CMS e-commerce in particular.

Another important finding was that using the proposed model, this study was able to gain insights into the quality of each CMS e-commerce websites investigated in the case study. The comparative analysis on the results found that there was no CMS with absolute quality performance. Instead, each of which has its own strength, weakness and different features in term of its freedom of use, ease of deployment, performance and security. Therefore, the findings offer a practical recommendation for those who are seeking the appropriate CMS for building e-commerce application for their business. While acknowledging the contribution, this study does not claim the proposed quality model is complete. Rather, it offers an overarching framework from which researchers can infuse additional criteria or matrices and test the new model in their future studies.

\section{REFERENCES}

[1] R. Stockdale, C. Standing, "The barriers and benefits of the electronic market place environment for SMEs," 8th Pacific Asia Conference on Information Systems, Shanghai, China , pp 1440-1452, 8-1 1 July 2004

[2] A.N.H. Zaied, "Barriers to e-commerce adoption in Egyptian SMEs," Int. J. Inf. Engineering Elect. Bussiness, vol 4 (3), pp 9, 2012

[3] L.A. Lefebvre, E. Lefebvre, "E-commerce and virtual enterprises: issues and challenges for transition economies", Technovation, vol 22 (5), pp 313-323, 2002

[4] E. Turban, D. King, J.K. Lee, T-P. Liang, D.C. Turban, "Ecommerce: mechanisms, platforms, and tools," Electronic Commerce: Springer, pp51-99, 2015

[5] N. Mehta, Choosing an Open Source CMS: Beginner's Guide: Packt Publishing Ltd; 2009.

[6] Guide D. CMS comparison 2018: The 5 most popular open source systems 2018 [cited 201828 August 2018]. Available from: https://www. 1 and1 .com/digitalguide/hosting/cms/cms-comparison-areview-of-the-five-best-platforms/.

[7] Record 25010II. Systems and Software Engineering - Systems and Software Quality Requirements and Evaluation (SQuaRE) - System and Soft ware Quality Models. Geneva: International Organisation for Standardisation; 2011.

[8] B. Behkamal, M. Kahani, M.K. Akbari, "Customizing ISO 9126 quality model for evaluation of B2B applications," Inf. Software Tech., vol 51(3), pp 599-609, 2009

[9] R.S. Pressman, Software engineering: a practitioner's approach: Palgrave Macmillan; 2005

[10] R. Florea, V. Stray, editors. Software Tester, We Want to Hire You! an Analysis of the Demand for Soft Skills2018; Cham: Springer International Publishing.

[11] A. Adewumi, S. Misra, N. Omoregbe, B. Crawford, R. Soto, A systematic literature review of open source software quality assessment models, SpringerPlus vol 5(1), pp 1936, 2016

[12] J.P. Miguel, D. Mauricio, G. Rodríguez, A review of software quality models for the evaluation of software products. arXiv preprint arXiv:14122977, 2014

[13] D.L. Simpson, editor Content for one: developing a personal content management system. Proceedings of the 33rd annual ACM SIGUCCS conference on User services, 2005: ACM.

[14] S.K. Patel, V. Rathod, J.B. Prajapati, "Performance analysis of content management systems-joomla, drupal and wordpress" Int. J. Computer Appl., vol 21(4), pp 39-43, 2011

[15] K-J. Stol, M.A. Babar, editors. A comparison framework for open source soft ware evaluation methods. IFIP International Conference on Open Source Systems; 2010: Springer.

[16] K.N. Malanga, J. Mehat, G. Ivaylo, F. Wabwoba, Assessing quality of open source software based on community metrics. 2015.

[17] S.S. Bahamdain, "Open Source Software (OSS) Quality Assurance: A Survey Paper," Procedia Computer Science, vol 56, pp 459-64, 2015

[18] A.A.A. Rasheed, S.D. El-Masri, "Comparing e-commerce content management systems" J. Theoretical Applied Inf. Tech., vol 53(3), 2013

[19] H. Wang, X. Li, B. Shi, Y. Zhang, W. Liu, "The Software Quality Management of Information System", Proc. 2nd Int. Conf. on Computer Sci. Electronics Engineering: Atlantis Press, 2013

[20] D.A. Wheeler, Generally recognized as mature (GRAM) OSS/FS programs 2006 [cited 201715 July 2017]. Available from: https://www.dwheeler.com/gram.html.

[21] C. Dorota, "Why do users choose Open Source software? Analysis of the net work effect," Informatyka Ekonomiczna, vol 1 (39), pp 9-22, 2016 